\title{
AN IMAGING VECTOR MAGNETOGRAPH FOR THE NEXT SOLAR MAXIMUM
}

\author{
Richard C. Canfield and Donald L. Mickey \\ Institute for Astronomy, University of Hawaii \\ Honolulu, Hawaii 96822
}

\begin{abstract}
.
Measurements of the vector magnetic field in the sun's atmosphere with high spatial and temporal resolution over a large field of view are critical to understanding the nature and evolution of currents in active regions. Such measurements, when combined with the thermal and nonthermal X-ray images from the upcoming Solar-A mission, will reveal the large-scale relationship between these currents and sites of heating and particle acceleration in flaring coronal magnetic flux tubes.

We describe the conceptual design of a new imaging vector magnetograph that combines a modest solar telescope with a rotating quarter-wave plate, an acousto-optical tunable prefilter as a blocker for a servo-controlled Fabry-Perot etalon, CCD cameras, and a rapid digital tape recorder. Its high spatial resolution ( $1 / 2$ arcsec pixel size) over a large field of view ( 4 by 5 arcmin) will be sufficient to significantly measure, for the first time, the magnetic energy dissipated in major solar flares. Its millisecond tunability and wide spectral range $(5000-8000 \AA)$ enable nearly simultaneous vector magnetic field measurements in the gas-pressure-dominated photosphere and magneticallydominated chromosphere, as well as effective co-alignment with Solar-A's X-ray images.
\end{abstract}




\section{PERFORMANCE CHARACTERISTICS}

- Spatial resolution: one arcsec. Detector pixel spacing of approximately 0.5 arcsec over a $4 \times 5$ arcmin field of view. This high resolution will critically sample the high quality image typical at Mees early in the day.

- Spectral resolution: $70 \mathrm{~m} \AA$ at $6000 \AA$. This resolution is marginal for the narrowest lines; at least three spectral samples will be required in the simplest cases, and probably quite a few more will be used for the standard program.

- Spectral range: 5000 - $\mathbf{6 5 0 0} \AA$. This range includes both photospheric (e.g. Fe I $\lambda 6302$ ) and chromospheric (e.g. Mg I $\lambda 5173)$ lines whose use for vector magnetic field measurement is well understood.

- Temporal resolution: A complete magnetogram in a single line in 15 seconds. This resolution is determined primarily by the data recording speed; better resolution can be achieved over a smaller field of view.

- Sensitivity: 10 Gauss longitudinal fields and 200 Gauss transverse fields in a few seconds. Simultaneous velocity measurements to $10 \mathrm{~m} / \mathrm{s}$. Temporal resolution can be traded for increased sensitivity.

- Co-alignment: A simultaneous photospheric white-light image of the full field of view, for precise co-alignment with Solar-A images and Max'91 ground and balloon-borne experiments. 


\section{DESIGN FEATURES}

- Telescope: 20-cm refractor. On-axis, as shown in the Figures 4 and 5. On the spar at Mees Solar Observatory, Haleakala, Maui.

- Monochromator: Air-spaced tunable Fabry-Perot, $70 \mathrm{~m} \AA$ bandpass. Order-sorting using an acousto-optic tunable filter (AOTF) with bandpass of $2 \AA$, a contrast of 1000:1, a large field of view, rapidly tunable over the full wavelength range.

- Polarization Modulator: Rotating quarter-wave plate. The AOTF will double as a beam-splitting analyzer.

- Detectors: High-resolution commercial CCD cameras. No mechanical shutter is necessary; turning off the radiofrequency signal to the AOTF turns off the diffracted beams imaged on the cameras. $754 \times 488$ pixel detector arrays.

- Data Acquisition: 68020-based computer in a VME-bus chassis. A minimum modulation sequence consists of a half-rotation of the wave plate, i.e. eight camera reads, which are combined to derive Stokes parameters. Recording on $8 \mathrm{~mm}$ digital video cassettes.

- Analysis and Archiving: Off-line analysis on a Sun workstation. A.rchival medium is the original $8 \mathrm{~mm}$ video cassette. Digital optical disk for archiving working datasets. Video disk recorder for time-dependence studies. 


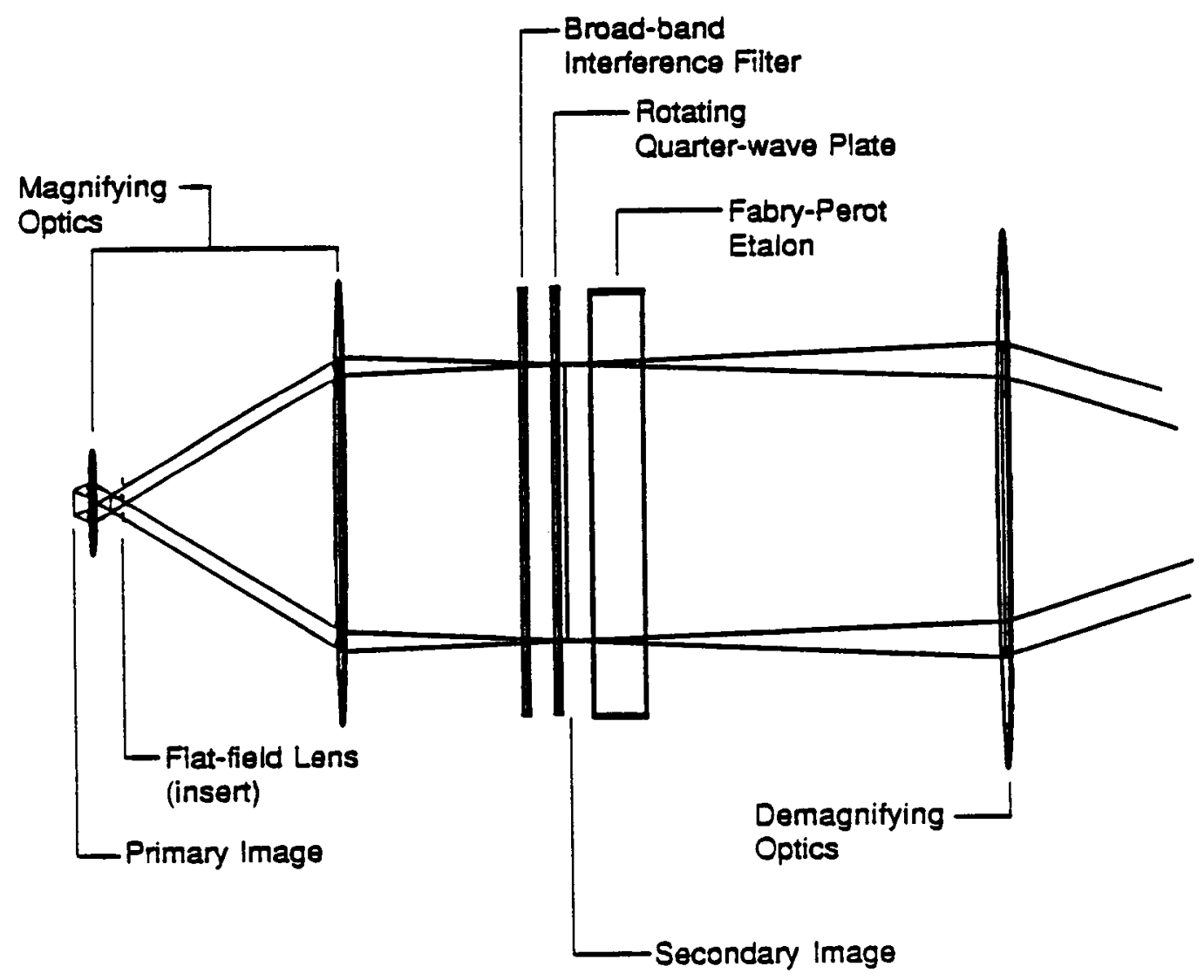

Figure 1. The modulator section of the Imaging Vector Magnetograph. Vertical exaggeration is s:1.

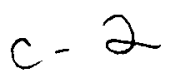




\section{Imaging Vector Magnetograph}

\section{Analyzer Section}

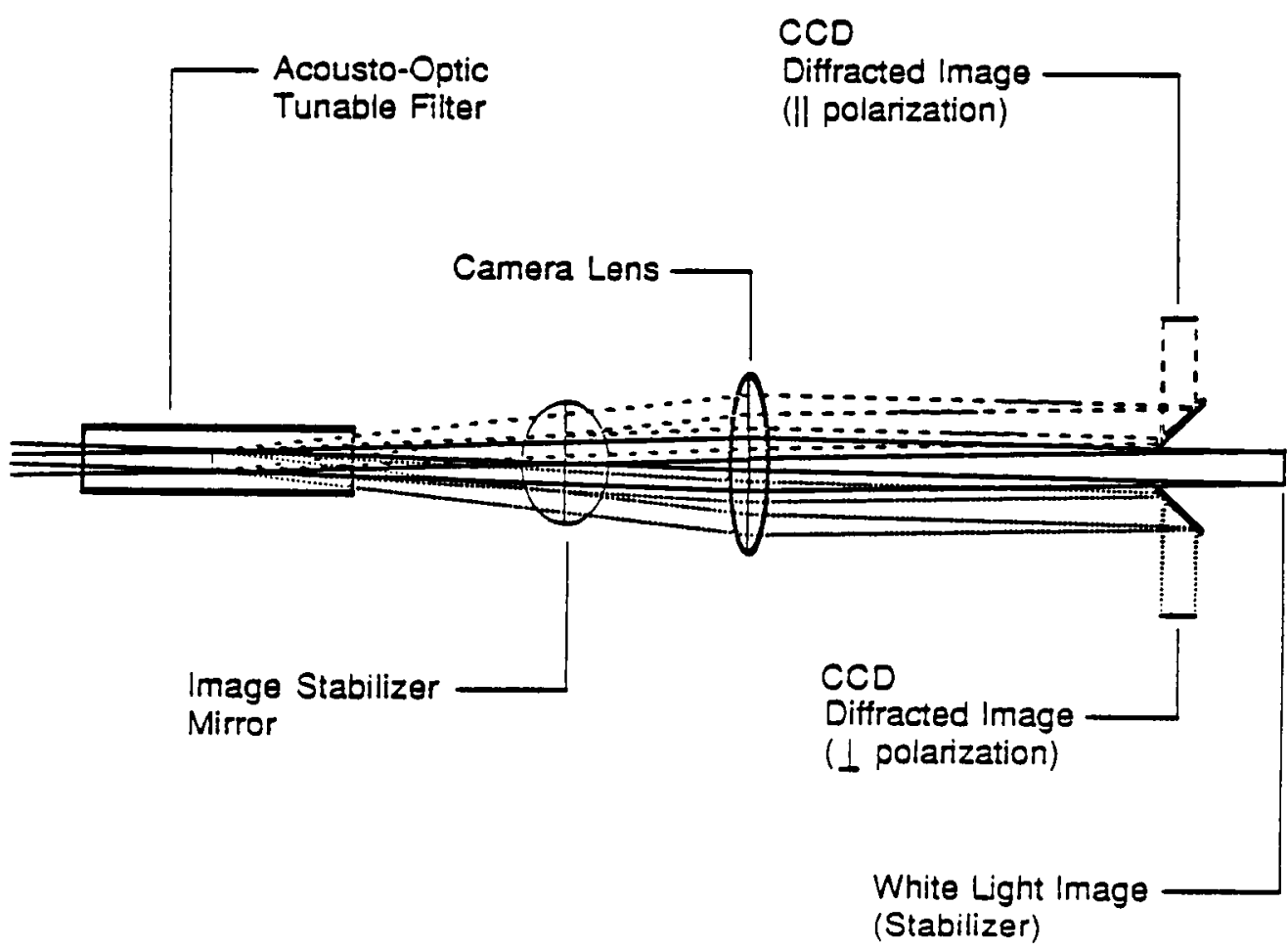

Figure 2. The analyzer section of the Imaging Vector Magnetograph. 